University of Nebraska - Lincoln

DigitalCommons@University of Nebraska - Lincoln

$5-15-2000$

\title{
The melting temperature of proton-disordered hexagonal ice: A computer simulation of 4-site transferable intermolecular potential model of water
}

\author{
G.T. Gao \\ University of Nebraska-Lincoln \\ Xiao Cheng Zeng \\ University of Nebraska-Lincoln, xzeng1@unl.edu \\ Hideki Tanaka \\ Okayama University, htanakaa@cc.okayama-u.ac.jp
}

Follow this and additional works at: https://digitalcommons.unl.edu/chemzeng

Part of the Chemistry Commons

Gao, G.T.; Zeng, Xiao Cheng; and Tanaka, Hideki, "The melting temperature of proton-disordered hexagonal ice: A computer simulation of 4-site transferable intermolecular potential model of water" (2000). Xiao Cheng Zeng Publications. 51.

https://digitalcommons.unl.edu/chemzeng/51

This Article is brought to you for free and open access by the Published Research - Department of Chemistry at DigitalCommons@University of Nebraska - Lincoln. It has been accepted for inclusion in Xiao Cheng Zeng Publications by an authorized administrator of DigitalCommons@University of Nebraska - Lincoln. 


\title{
The melting temperature of proton-disordered hexagonal ice: A computer simulation of 4-site transferable intermolecular potential model of water
}

\author{
G. T. Gao a) and X. C. Zeng \\ Department of Chemistry, University of Nebraska-Lincoln, Lincoln, Nebraska 68588 \\ Hideki Tanaka \\ Department of Chemistry, Okayama University, Okayama 700-8530, Japan
}

(Received 21 January 2000; accepted 17 February 2000)

\begin{abstract}
We report computer simulation results of free energies of proton-disordered hexagonal ice and liquid water at atmospheric pressure with the 4-site transferable intermolecular potential model of water. A new reference system is used to calculate the free energy of the ice phase. The melting point of proton-disordered hexagonal ice at atmospheric pressure is found to be $T_{m}=238$ $( \pm 7) \mathrm{K}$. This result is consistent with a previous estimation, $230 \mathrm{~K}<T_{m}<250 \mathrm{~K}$, from molecular dynamic simulation of the surface melting of the proton-disordered hexagonal ice [G.-J. Kroes, Surf. Sci. 275, 365 (1992)]. (C) 2000 American Institute of Physics.
\end{abstract} [S0021-9606(00)50618-7]

\section{INTRODUCTION}

Among simple rigid models of water developed to date $^{1,2}$ the simple point charge (SPC) model and the extended simple point charge (SPC/E) model of Berendsen et al., ${ }^{3,4}$ as well as the 4-site transferable intermolecular potential (TIP4P) model of Jorgensen et al., ${ }^{5}$ have been the popular choices in many computer simulations of water over the past two decades. These pair-additive potential models of water are parametrized to reproduce the measured properties of bulk water at ambient temperature and pressure. Although the latest effort in parametrizing water potentials has been directed towards non-pair-additive polarizable models with the hope for a unified description of water clusters, bulk phases (liquid water, supercritical water, and ice), and interfaces, ${ }^{6-8,2}$ it seems the simple pair-additive water models can still be useful for quite a while, particularly to provide physical insight in problems related to normal ice/water interface, ${ }^{9,10}$ normal ice surface, ${ }^{11-13}$ slow dynamics of supercooled water, ${ }^{14,15}$ and the possible low density to high density liquid phase transition in supercooled water. ${ }^{16,17}$ Common to all these problems is the presence of the normal ice or the supercooled liquid phase. It is therefore desirable to know exactly the melting point of the proton-disordered hexagonal ice (normal ice) at atmospherical pressure with the simple water models. The focus of this paper is to determine the melting point of the TIP4P model of normal ice.

To our knowledge, Karim and Haymet were the first to study the melting point of TIP4P ice. ${ }^{9}$ From molecular dynamics simulations of proton-ordered hexagonal ice-water interfaces, Karim and Haymet found the melting point of TIP4P ice has an upper bound of $270 \mathrm{~K}$. Nada and Furukawa ${ }^{10}$ also investigated proton-ordered hexagonal icewater interfaces and concluded that the melting point of

a)Electronic mail: gao@phase1a.unl.edu
TIP4P ice is higher than $230 \mathrm{~K}$. However, recent free-energy calculations by Vlot et al. ${ }^{18}$ show that the melting point of proton-ordered hexagonal ice is $214( \pm 6) \mathrm{K}$, much lower than $230 \mathrm{~K}$. Vlot et al.'s calculations clearly demonstrate that the most reliable way to locate the melting point is to obtain the absolute free energies of both TIP4P ice and liquid phases. For proton-disordered hexagonal TIP4P ice, it appears that the only computer simulation study of melting point was done by Kroes. ${ }^{11}$ Kroes found from molecular dynamics simulations of the surface melting of TIP4P ice that the melting point is within the range of 230 and $250 \mathrm{~K}$. In this paper, we will show that our free-energy calculations support Kroes' estimation. We find the melting point of the TIP4P ice is $238( \pm 7) \mathrm{K}$.

\section{MODEL AND METHOD}

The TIP4P model of water ${ }^{5}$ describes a single water molecule by four rigid points with an $\mathrm{OH}$ distance of 0.9572 $\AA$ and an $\mathrm{HOH}$ angle $104.52^{\circ}$. Two positive point charges are placed on $\mathrm{H}$ atoms and their magnitudes are $q_{H}$ $=0.52 e$, a negative point charge with magnitude $q=-2 q_{H}$ $=-1.04 e$ is placed on a point $0.15 \AA$ away from the $\mathrm{O}$ atom, along the water bisector. Only the $\mathrm{O}$ atoms interact among themselves through a Lennard-Jones potential, with the energy parameter $\epsilon_{\mathrm{O}}=0.649 \mathrm{~kJ} / \mathrm{mol}$ and size parameter $\sigma_{\mathrm{O}}=3.154 \AA$. Following a previous work, ${ }^{19}$ a switching function is used to smoothly shift the pairwise potential function to zero.

To calculate the free energy of both ice and liquid phases, we employ the thermodynamic integration method. ${ }^{20,21}$ This method has been used successfully in freeenergy calculations for atomic ${ }^{20,22}$ and orientationally disordered molecular systems. ${ }^{23}$ In this method, computer simulations are carried out for a system with the potential energy function of the form

$$
U(\lambda)=U_{0}+\lambda^{k}\left(U_{1}-U_{0}\right),
$$


where $U_{0}$ is the potential energy function of a simple reference system whose free energy can be evaluated analytically and $U_{1}$ is the potential energy function of the system to be studied. In Eq. (1), $k$ is generally a positive integer and $\lambda$ is a coupling parameter for which $\lambda=0$ corresponds to the reference system and $\lambda=1$ corresponds to the system to be studied. In the simulation, the values of $\langle\partial U(\lambda) / \partial \lambda\rangle_{N, V, T, \lambda}$ from $\lambda=0$ to $\lambda=1$ are calculated, which give rise to the Helmholtz free-energy difference of the two systems

$$
\Delta A=\int_{0}^{1}\left\langle\frac{\partial U(\lambda)}{\partial \lambda}\right\rangle_{N, V, T, \lambda} d \lambda,
$$

where \langle\rangle$_{N, V, T, \lambda}$ denotes the ensemble average is under fixed number of molecules $N$, volume $V$, temperature $T$, and the coupling parameter $\lambda$. Conventionally, the reference system for the liquid and crystal phase is chosen to be the ideal gas and the harmonic Einstein crystal with the same crystal structure, respectively.

Recently, the thermodynamic integration method has been extended to treat the molecular systems with orientational order in the crystal phase such as ice. ${ }^{18,24}$ In the extended method, the harmonic Einstein crystal is still used as the reference system to constrain the long-range translational order of the crystal. Moreover, several functions of Euler angles are also introduced in the potential function to constrain the long-range orientational order. Therefore, several force constants are needed in the extended method. Note that the values of force constants can be chosen arbitrarily because in principle the final results of free energy of a crystal should not depend on the choice of the force constants. Once the free energies of both liquid and solid phases as a function of temperature are known, the melting point is determined from the crossing point of the free-energy curves. However, for proton-disordered ice, many configurations of ice are needed to calculate the average free energy. As a result, we find that for the proton-disordered ice the determination of the melting point is sensitive to the choice of the force constants because a small numerical change in the free energy of ice can lead to a large variation of the location of melting point.

In light of this sensitivity of melting point to the accuracy of free-energy calculation, we propose to use an alternative reference system to calculate the free energy of the ice. The proposed reference system for ice crystal is the Debye model of crystal. It is well-known that the Debye model can describe the thermodynamic properties of crystals more realistically. For example, the Debye model predicts the well-known $T^{3}$ law of the heat capacity for crystal at low temperatures, as found in experiments. It turns out that evaluation of the free energy based on the Debye crystal reference system is actually computationally more efficient than searching for a set of optimal force constants for harmonic Einstein crystal. Another nice feature is that for the Debye model the force constants can be uniquely determined from the normal-mode calculation.

In practice, the potential energy function $U_{1}$ of the molecular crystal is divided into two parts: a harmonic part $U^{h}$ and an anharmonic part $U^{a}$, that is,

$$
U_{1}=U^{h}+U^{a} .
$$

The harmonic part can be treated naturally in the framework of the Debye model. Indeed, the harmonic part can be viewed as a collection of normal-mode harmonic oscillators. As a result, $\left\langle U^{h}\right\rangle_{N, V, T}$ for $6 N$ nonlinear rigid rotors can be calculated via the equation

$$
\left\langle U^{h}\right\rangle_{N, V, T}=U_{q}+3 N k_{B} T,
$$

where $U_{q}$ is the potential energy of the local minimum structure at a given density and $k_{B}$ is Boltzmann's constant. The normal-mode frequency spectrum or the density of state for intermolecular vibrations $g(\omega)$ ( $\omega$ denotes the frequency of normal modes) is obtained by means of normal-mode analysis, ${ }^{25,26,19}$ or more specifically, by diagonalizing a matrix of the second-order derivatives of the potential energy with respect to the mass-weighted coordinates. Once $g(\omega)$ is known, the Helmholtz free energy of the Debye crystal can be calculated by

$$
A^{h}=U_{q}+6 N k_{B} T \int_{0}^{\infty} g(\omega) \log \frac{h \omega}{k_{B} T} d \omega .
$$

With the harmonic Debye crystal as the reference system, the anharmonic part of Helmholtz free energy of the molecular crystal $\left(A^{a}\right)$ is essentially the $\Delta A$ term in Eq. (2), which can be calculated straightforwardly via the thermodynamic integration method. It should be noted that $A^{a}$ vanishes approaching $T=0$.

For the proton-disordered hexagonal ice, the $U_{0}$ term in Eq. (2) is chosen to be $U^{h}$ and $k=2$. Thus,

$$
U(\lambda)=U^{h}+\lambda^{2}\left(U_{1}-U^{h}\right),
$$

where $U_{1}$ represents the potential energy function described by the full TIP4P potential of water. The anharmonic part of Helmholtz free energy is then given by

$$
A^{a}=\int_{0}^{1} 2 \lambda\left\langle U_{1}-U^{h}\right\rangle_{N, V, T, \lambda} d \lambda .
$$

For the liquid water, on the other hand, the ideal gas of rigid nonlinear molecules is generally chosen as the reference system. Thus, in Eq. (1), $U_{0}=0$. In addition, $k=4$ is chosen here for calculating the free energy of liquid water. Note that, for the ideal gas, the Helmholtz free energy can be written in an analytical form

$$
\begin{aligned}
A^{\mathrm{id}=} & N k_{B} T\left(\log \rho-1+\log \Lambda^{3}-\log \left[\frac{\pi^{1 / 2}}{\sigma}\left(\frac{8 \pi^{2} I_{A} k_{B} T}{h^{2}}\right)^{1 / 2}\right.\right. \\
& \left.\left.\times\left(\frac{8 \pi^{2} I_{B} k_{B} T}{h^{2}}\right)^{1 / 2}\left(\frac{8 \pi^{2} I_{C} k_{B} T}{h^{2}}\right)^{1 / 2}\right]\right),
\end{aligned}
$$

where $\rho$ is the density of liquid water, $\Lambda$ is the thermal wave length, $I_{A}, I_{B}$, and $I_{C}$ are the principal moments of inertia of a TIP4P water molecule, and the symmetry number $\sigma$ is 2 . The free energy difference from the ideal gas, namely the excess Helmholtz free energy, can then be calculated from Eq. (2). Once the Helmholtz free energy is known, the Gibbs free-energy $G$ can be calculated by $G=A+p V$, where $p$ is the pressure and $V$ is the volume of the system. 


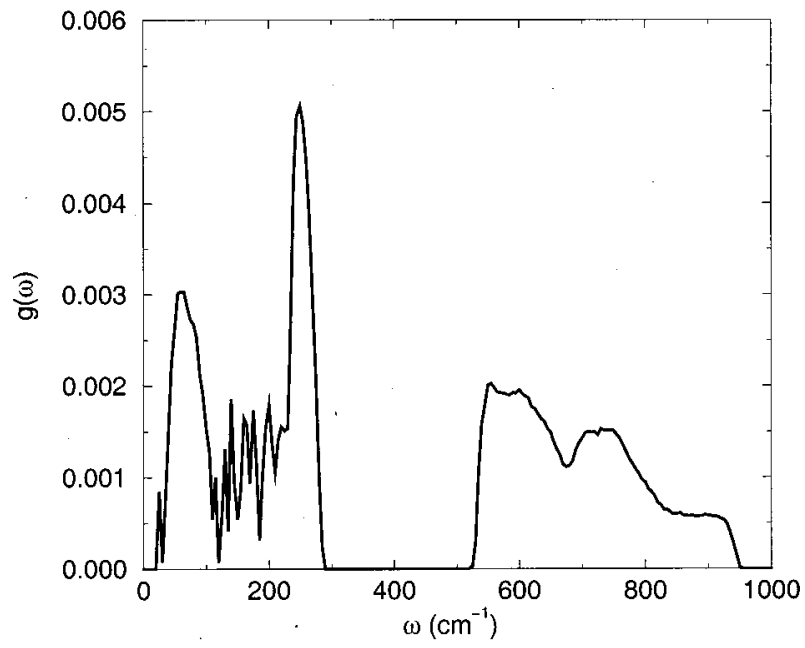

FIG. 1. Density of state of the proton-disordered hexagonal ice averaged over five configurations at $T=255 \mathrm{~K}$ and $p=1 \mathrm{~atm}$.

\section{SIMULATION RESULTS}

Standard Metropolis Monte Carlo simulations are carried out for both the solid and liquid states of water. Periodic conditions are employed all the time. Five proton-disordered hexagonal ice configurations are used in the free-energy calculations. Each configuration contains 288 and 256 TIP4P water molecules for hexagonal ice and liquid water, respectively. The equilibrium structures and densities are obtained from isobaric Monte Carlo simulation at atmospherical pressure $p=1 \mathrm{~atm}$ and for a series of temperature ranging from 200 to $290 \mathrm{~K}$. For every equilibrate ice structure, the steepest descent method is used to quench the system to a local minimum of the total potential energy $U_{q}$. The corresponding configuration is called an inherent structure introduced by Stillinger and Weber. ${ }^{27}$ Normal-mode analysis is then followed for every inherent structure to obtain the frequency spectra $g(\omega)$. Figure 1 shows a normal-mode spectrum for hexagonal ice averaged over five configurations. As can be seen, the normal modes are distributed mainly in two regions: one from 25 to $290 \mathrm{~cm}^{-1}$ and another from 520 to 950 $\mathrm{cm}^{-1}$. The former is associated with translational motions and the latter with rotational motions of individual molecules. The spectrum in the lower frequency region is composed of two main peaks. One peak corresponds to a bending motion of three hydrogen-bonded molecules and the other is associated with a stretching motion of a hydrogen-bonded pair. This feature is different from the case of simple liquid, where only a single broad peak is observed. It is useful to have more insight on the characters of local modes involving a few water molecules mutually hydrogen bonded. For this purpose, we pick up a central molecule arbitrarily and choose $(n-1)$ molecules out of the neighboring 4 molecules. We examine the vibrational modes for $n=1,2,3$, and 5 by diagonalizing the $6 n \times 6 n$ submatrices. We find that for $n=2$ the bending motions may show up but they become more notable for $n=4$. In Fig. 2 we only show the densities of state for $n=1$ and 5 to compare with that of the whole system. It can be seen that the spectrum for $n=5$ has some

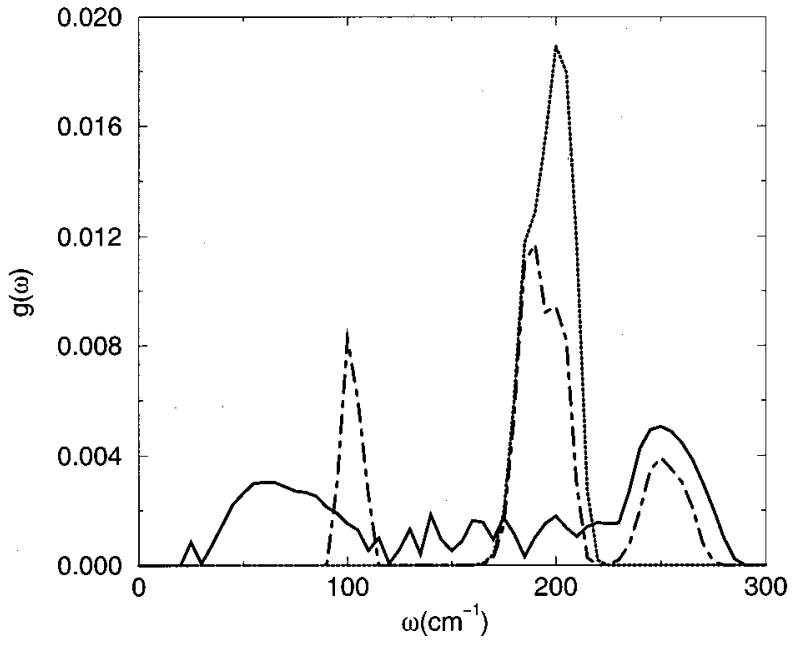

FIG. 2. Densities of state for intermolecular vibrational motions of the whole system (solid line), a monomer (dotted line), and a pentamer (dashdot line) in the proton-disordered hexagonal ice.

resemblance to that of the whole system. In the case of a single molecule, $n=1$ (Einstein model), the spectrum does not cover the lower frequency region that corresponds to the bending motions.

In Fig. 3 we show a typical $\langle\partial U(\lambda) / \partial \lambda\rangle_{N, V, T, \lambda}$ versus $\lambda$ curve for the ice at temperature $T=255 \mathrm{~K}$. Free energies of the proton-disordered hexagonal ice are computed for temperatures from $T=200$ to $290 \mathrm{~K}$ with an increment of $5 \mathrm{~K}$. Results are given in Table I. Since the ice is proton disordered, another contribution to the total free energy, namely the residual configuration entropy due to the proton disorder, must be included. This configuration entropy can be accurately given by the equation, $-T S_{0}=-N k_{B} T \log (3 / 2)$, derived by Pauling. ${ }^{28}$ From Table I, one can see that the anharmonic part of free energies is always negative. This is sensible because the anharmonic interactions always drive the system to more disordered configurations, and thus increase the entropy of the system.

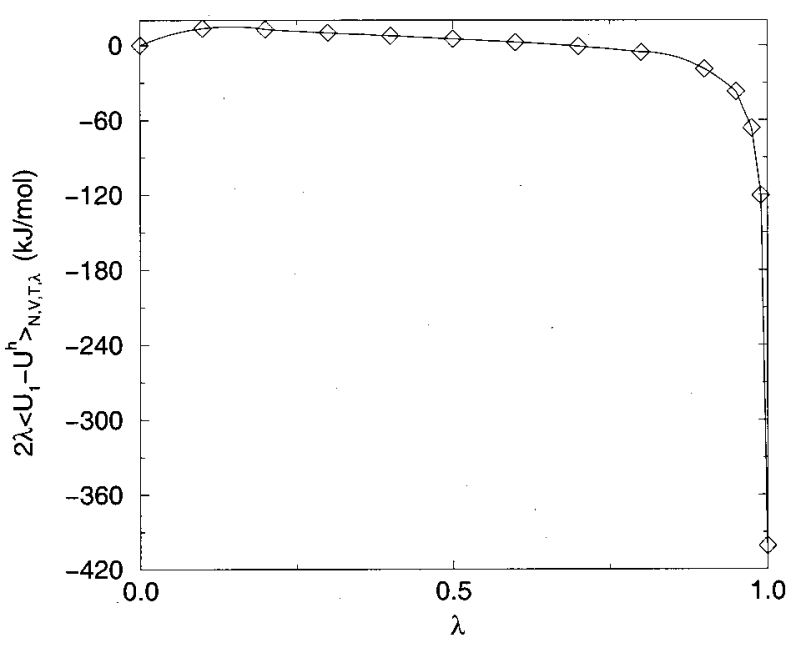

FIG. 3. $\langle\partial U(\lambda) / \partial \lambda\rangle_{N, V, T, \lambda}$ versus $\lambda$ curve for the proton-disordered hexagonal ice at $T=255 \mathrm{~K}$ and $p=1 \mathrm{~atm}$. 
TABLE I. Calculated harmonic $\left(A^{h}\right)$, anharmonic $\left(A^{a}\right)$, and total Helmholtz free energies $(A)$ of the proton-disordered hexagonal TIP4P ice as a function of temperature $T$ from 200 to $290 \mathrm{~K}$.

\begin{tabular}{cccc}
\hline \hline$T(\mathrm{~K})$ & $A^{h}(\mathrm{~kJ} / \mathrm{mol})$ & $A^{a}(\mathrm{~kJ} / \mathrm{mol})$ & $A(\mathrm{~kJ} / \mathrm{mol})$ \\
\hline 200 & -48.58 & $-1.31 \pm 0.13$ & -49.89 \\
205 & -48.64 & $-1.39 \pm 0.11$ & -50.03 \\
210 & -48.71 & $-1.32 \pm 0.11$ & -50.03 \\
215 & -48.79 & $-1.37 \pm 0.04$ & -50.16 \\
220 & -48.87 & $-1.27 \pm 0.09$ & -50.14 \\
225 & -48.95 & $-1.19 \pm 0.10$ & -50.14 \\
230 & -49.04 & $-1.54 \pm 0.17$ & -50.58 \\
235 & -49.15 & $-1.32 \pm 0.07$ & -50.47 \\
240 & -49.25 & $-1.32 \pm 0.09$ & -50.57 \\
245 & -49.35 & $-1.56 \pm 0.07$ & -50.91 \\
250 & -49.48 & $-1.43 \pm 0.16$ & -50.91 \\
255 & -49.60 & $-1.60 \pm 0.15$ & -51.20 \\
260 & -49.72 & $-1.82 \pm 0.10$ & -51.54 \\
265 & -49.85 & $-1.82 \pm 0.06$ & -51.67 \\
270 & -49.95 & $-1.77 \pm 0.15$ & -51.72 \\
275 & -50.12 & $-1.72 \pm 0.16$ & -51.84 \\
280 & -50.22 & $-1.89 \pm 0.04$ & -52.11 \\
285 & -50.38 & $-2.28 \pm 0.11$ & -52.66 \\
290 & -50.10 & $-2.80 \pm 0.16$ & -52.90 \\
\hline \hline
\end{tabular}

To assess the accuracy of the present free-energy calculations with the Debye crystal as the reference, we also carried out free-energy calculations with the conventional harmonic Einstein crystal as the reference. We employed a reference potential energy function as used in Ref. 18,

$U_{0}=\sum_{i}\left[C_{R}\left|\mathbf{r}_{\mathbf{i}}-\mathbf{R}_{\mathbf{i}}\right|^{2}+C_{\theta}\left(1-\cos \theta_{i}\right)+C_{\omega}\left(1-\cos 2 \omega_{i}\right)\right]$,

where $\mathbf{r}_{\mathbf{i}}$ is the coordinate of the center of mass of the molecule, $\mathbf{R}_{\mathbf{i}}$ is the coordinate of the lattice site, and $\theta_{i}$ and $\omega_{i}$ are two Euler angles of the water molecule. We arbitrarily chose three sets of parameters for the force constants $C_{R}$, $C_{\theta}$, and $C_{\omega}$. These parameters and the corresponding calculated free energies of the ice are listed in Table II. It can be seen from Table II that the calculated free energies change slightly with different sets of the force constants. Even though these changes are only a few tenths of $1 \mathrm{~kJ} / \mathrm{mol}$ (about 1 percent in relative error), it turns out that they can lead to a shift in the location of melting point by more than $10 \mathrm{~K}$. Note also that the free energies shown in Table II are all slightly higher than the calculated free energy with the Debye crystal as reference. The smallest difference is 0.37 $\mathrm{kJ} / \mathrm{mol}$, which is still a little larger than the typical statistical

TABLE II. Calculated reference $\left(A_{0}=A_{E}+U_{q}\right)$ and total Helmholtz free energies $\left(A=A_{0}+\Delta A\right)$ at $T=255 \mathrm{~K}$ where $A_{E}$ is the free energy of the harmonic Einstein crystal.

\begin{tabular}{cccccc}
\hline \hline $\begin{array}{c}C_{R} / k_{B} T \\
\left(\AA^{-2}\right)\end{array}$ & $C_{\theta} / k_{B} T$ & $C_{\omega} / k_{B} T$ & $\begin{array}{c}A_{0} \\
(\mathrm{~kJ} / \mathrm{mol})\end{array}$ & $\begin{array}{c}\Delta A \\
(\mathrm{~kJ} / \mathrm{mol})\end{array}$ & $\begin{array}{c}A \\
(\mathrm{~kJ} / \mathrm{mol})\end{array}$ \\
\hline 65.0 & 250.0 & 35.0 & -44.55 & -6.28 & -50.83 \\
50.0 & 150.0 & 35.0 & -46.47 & -4.22 & -50.69 \\
30.0 & 50.0 & 25.0 & -50.78 & 0.55 & -50.23 \\
\hline \hline
\end{tabular}

TABLE III. Coefficients in the polynomial $H=a_{0}+a_{1} T+a_{2} T^{2}+a_{3} T^{3}$ used as a fit to the enthalpies of water at a function of temperature $T$ from 200 to $300 \mathrm{~K}$. The enthalpy is in units of $\mathrm{kJ} / \mathrm{mol}$.

\begin{tabular}{cccc}
\hline \hline$a_{0}$ & $a_{1}$ & $a_{2}$ & $a_{3}$ \\
\hline-49.77939 & -0.04290033 & $4.914896 \times 10^{-4}$ & $-5.871010 \times 10^{-7}$ \\
\hline \hline
\end{tabular}

error $0.1 \mathrm{~kJ} / \mathrm{mol}$. To reduce the free-energy difference within the statistical error bar, it seems a search for an optimal set of force constants is needed.

In order to pin down the melting point of the protondisordered hexagonal ice, we also calculate the free energies of TIP4P liquid water as a function of temperature. First, an isobaric Monte Carlo simulation is carried out to obtain the density of the liquid water, which is $\rho_{0}=0.994 \mathrm{~g} / \mathrm{cm}^{3}$, at $p_{0}=1 \mathrm{~atm}$ and $T_{0}=300 \mathrm{~K}$. Next, at the same density and temperature, the Helmholtz free energy is calculated using the thermodynamic integration method with the ideal gas as the reference system. The obtained Helmholtz free energy and the quantity $p_{0} V$ give rise to the Gibbs free energy at this state, $G_{0}=-54.04 \mathrm{~kJ} / \mathrm{mol}$. Another series of isobaric Monte Carlo simulations is then carried out at $p_{0}=1 \mathrm{~atm}$ and various temperature ranging from 195 to $295 \mathrm{~K}$. The enthalpies $H=U+p_{0} V$ at these temperatures are obtained and fitted to a simple polynomial. Coefficients of the polynomial are listed in Table III. Finally, the temperature dependence of the Gibbs free energy under the isobaric condition $\left(p_{0}\right.$ $=1 \mathrm{~atm})$ is evaluated using the Gibbs-Helmholtz equation

$$
\frac{G}{T}-\frac{G_{0}}{T_{0}}=-\int_{T_{0}}^{T} \frac{H}{T^{2}} d T .
$$

In Fig. 4, we show the Gibbs free energies per mole as a function of temperature for both liquid and the protondisordered hexagonal ice. From the crossing point of the two free-energy curves, we find that the melting temperature of

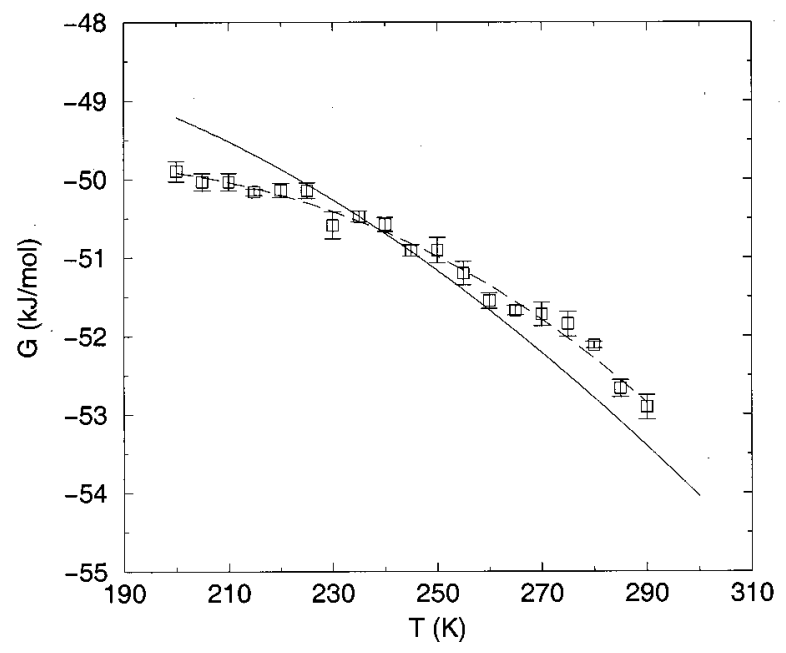

FIG. 4. Temperature dependence of the Gibbs free energies for the liquid water (solid line) and the proton-disordered hexagonal ice (squares with error bars). The dashed line is a fit to the free energies of the ice. The crossing point of the solid and dashed lines gives the value of the melting point at the atmospheric pressure. 
the proton-disordered hexagonal TIP4P ice is $T_{m}=238$ $( \pm 7) \mathrm{K}$. This estimation is consistent with the result of Kroes, ${ }^{11}$ who investigated the surface melting of the protondisordered hexagonal ice using molecular dynamics simulations. He found the melting point of the ice is between 230 and $250 \mathrm{~K}$. Also, it can be seen in Fig. 3 that the slopes of both free-energy curves are negative in the temperature range examined, which means the entropies $S$ are always positive as $S=-(\partial G / \partial T)_{P}$.

\section{CONCLUDING REMARKS}

Free-energy calculations of both solid and liquid phases are necessary to determine the location of the melting point. However, for the proton-disordered hexagonal TIP4P ice at atmospherical pressure, it is found that a change of 1 percent in the free energies of the proton-disordered ice can lead to a shift of the melting point by more than $10 \mathrm{~K}$. Thus, highly accurate free-energy calculations for the proton-disordered ice phase are needed to determine the exact location of the melting point. In view of this sensitivity of melting point to the accuracy of free-energy calculations, we propose to use the Debye model of crystal as an alternative reference system for the ice phase when applying the thermodynamic integration method. With this reference system the force constants can be uniquely determined from the normal-mode analysis. Free energy of the ice can be calculated accurately, yet without much more computational effort compared with using the harmonic Einstein crystal as the reference system.

From the calculated free energies of the protondisordered hexagonal ice and the liquid water, we find the melting point of TIP4P ice at atmospheric pressure is $T_{m}$ $=238( \pm 7) \mathrm{K}$, consistent with a previous estimation by Kroes from a molecular dynamic simulation of surface melting of the same ice structure and model of water. Compared with the melting temperature of real water $T_{m}=273 \mathrm{~K}$, the TIP4P model of water underestimates the melting temperature of normal ice by about $35 \mathrm{~K}$. Interestingly, a density of maximum in liquid TIP4P water is found to appear at about $258 \mathrm{~K},{ }^{29} 19 \mathrm{~K}$ below that of real water $(277 \mathrm{~K})$. Moreover, a previous Gibbs ensemble Monte Carlo calculation indicates that the TIP4P model of water also underestimates the critical point of real water by about several tens of kelvin. ${ }^{30}$ These systematic deviations from experimental values suggest that the TIP4P model of water, although parametrized from properties of bulk liquid at the ambient condition, can be of semiquantitative value to explore physical properties of ice, supercooled and glassy water, and ice/water interface.

\section{ACKNOWLEDGMENTS}

X.C.Z. thanks NSF, ONR, and JSPS for support of this work. H.T. is grateful to the Japan Ministry of Education and the computer center of IMS for support of this work.

${ }^{1}$ G. W. Robinson, S.-B. Zhu, S. Gingh, and M. W. Evans, Water in Biology, Chemistry and Physics: Experimental Overview and Computational Methodologies (World Scientific, Singapore, 1996).

${ }^{2}$ V. Buch, P. Sandler, and J. Sadlej, J. Phys. Chem. B 102, 8641 (1998).

${ }^{3}$ H. J. C. Berendsen, J. P. M. Postma, and W. F. van Gunsteren, Intermolecular Forces, edited by B. Pullman (Reidel, Dordrecht, 1981).

${ }^{4}$ H. J. C. Berendsen, J. R. Grigera, and T. P. Straatsma, J. Phys. Chem. 91, 6269 (1987).

${ }^{5}$ W. L. Jorgensen, J. Chandrasekhar, J. D. Madura, R. W. Impey, and M. L. Klein, J. Chem. Phys. 79, 926 (1983).

${ }^{6}$ A. A. Chialvo and P. T. Cummings, J. Chem. Phys. 105, 8274 (1996).

${ }^{7}$ S. J. Stuart and B. J. Berne, J. Phys. Chem. 100, 11934 (1996).

${ }^{8}$ L. X. Dang and T.-M. Chang, J. Chem. Phys. 106, 8149 (1997).

${ }^{9}$ O. A. Karim and A. D. J. Haymet, Chem. Phys. Lett. 138, 531 (1987).

${ }^{10}$ H. Nada and Y. Furukawa, J. Phys. Chem. B 101, 6163 (1997).

${ }^{11}$ G. J. Kroes, Surf. Sci. 275, 365 (1992).

${ }^{12}$ H. Nada and Y. Furukawa, Appl. Surf. Sci. 121/122, 445 (1997).

${ }^{13}$ V. Buch, L. Delzei, C. Blackledge, and J. P. Devlin, J. Phys. Chem. 100, 3732 (1996).

${ }^{14}$ P. Gallo, F. Sciortino, P. Tartaglia, and S.-H. Chen, Phys. Rev. Lett. 76, 2730 (1996).

${ }^{15}$ F. W. Starr, S. Harrington, F. Sciortino, and H. E. Stanley, Phys. Rev. Lett. 82, 3629 (1999).

${ }^{16}$ P. H. Poole, U. Essmann, F. Sciortino, and H. E. Stanley, Nature (London) 360, 324 (1992).

${ }^{17}$ H. Tanaka, Nature (London) 380, 328 (1996).

${ }^{18}$ M. J. Vlot, J. Huinink, and J. P. van der Eerden, J. Chem. Phys. 110, 55 (1999).

${ }^{19}$ I. Ohmine, H. Tanaka, and P. G. Wolynes, J. Chem. Phys. 89, 5852 (1988).

${ }^{20}$ D. Frenkel and A. J. C. Ladd, J. Chem. Phys. 81, 3188 (1984).

${ }^{21}$ D. Frenkel and B. Smit, Understanding Molecular Simulation (Academic, San Diego, 1996).

${ }^{22}$ B. B. Laird and A. D. J. Haymet, Mol. Phys. 75, 71 (1992).

${ }^{23}$ E. J. Meijer, D. Frenkel, R. A. LeSar, and A. J. C. Ladd, J. Chem. Phys. 92, 7570 (1990).

${ }^{24}$ L. A. Báez and P. Clancy, Mol. Phys. 86, 385 (1995).

${ }^{25}$ A. Pohorille, L. R. Pratt, R. A. LaViolette, M. A. Wilson, and M. A. MacElroy, J. Chem. Phys. 87, 6070 (1987).

${ }^{26}$ E. Bright, J. C. Decius, and P. C. Cross, Molecular Vibrations (Dover, New York, 1955).

${ }^{27}$ F. H. Stillinger and T. A. Weber, Science 225, 983 (1984).

${ }^{28}$ L. Pauling, J. Am. Chem. Soc. 57, 2680 (1935).

${ }^{29}$ W. L. Jorgensen and C. Jenson, J. Comput. Phys. 19, 1179 (1998).

${ }^{30}$ J. J. De Pablo and J. M. Prausnitz, Fluid Phase Equilibria 53, 177 (1989). 